\title{
Bootstrapping for Batch Active Sampling
}

\author{
Heinrich Jiang \\ heinrichj@google.com \\ Google Research \\ Mountain View, CA, USA
}

\author{
Maya R. Gupta \\ mayagupta@google.com \\ Google Research \\ Mountain View, CA, USA
}

\begin{abstract}
The goal of active learning is to select the best examples from an unlabeled pool of data to label to improve a model trained with the addition of these labeled examples. We discuss a real-world use case for batch active sampling that works at larger scales. The standard margin algorithm has repeatedly been shown difficult to beat in practice for the classic active sampling set-up, but for larger batches and candidate pools, we show that margin sampling may not provide enough diversity. We present a simple variant of margin sampling for the batch setting that scores candidate samples by their minimum margin to a set of bootstrapped margins, and explain how this proposal increases diversity in a supervised and efficient way, and why it differs from the usual ensemble methods for active sampling. Experiments on benchmark datasets show that the proposed min-margin sampling consistently works better than margin as the batch size grows, and better than the five other diversity-encouraging active sampling methods we tested. Two realworld case studies illustrate the practical value, and help highlight challenges of applying and deploying batch active sampling.
\end{abstract}

\section{CCS CONCEPTS}

- Computing methodologies $\rightarrow$ Bagging; Active learning settings.

\section{KEYWORDS}

active sampling, bootstrap, ensemble

\section{ACM Reference Format:}

Heinrich Jiang and Maya R. Gupta. 2021. Bootstrapping for Batch Active Sampling. In Proceedings of the 27th ACM SIGKDD Conference on Knowledge Discovery and Data Mining (KDD '21), August 14-18, 2021, Virtual Event, Singapore. ACM, New York, NY, USA, 11 pages. https://doi.org/10.1145/ 3447548.3467076

\section{PROBLEM STATEMENT}

Machine learning depends on having useful training examples. In practice, there is already some existing training data and a preliminary model. Then one may have a pool of unlabelled examples, and be able to select some of those examples to be labeled and added to the training set. The problem of deciding which candidates are the best bets to label is known as active sampling. The classic active

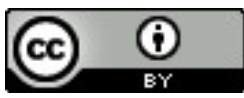

This work is licensed under a Creative Commons Attribution International 4.0 License.

KDD '21, August 14-18, 2021, Virtual Event, Singapore.

(c) 2021 Copyright held by the owner/author(s).

ACM ISBN 978-1-4503-8332-5/21/08.

https://doi.org/10.1145/3447548.3467076 sampling set-up chooses a single candidate example to label from a fixed pool of candidates, gets its label, re-trains the model, and repeats with the same fixed candidate pool.

For many of the larger machine-learned models we run in practice, this set-up does not match our machine-learning pipelines. A key difference from the classic set-up is that with today's flexible machine learning models, due to the randomness of training, we often need to add a relatively large batch of new labeled examples to produce a re-trained model that is measurably better than the original model [1], and worth the cost of possibly costly A/B tests and live experiments. Further, model training can be slow, especially if the training set is changing enough to warrant re-optimizing hyperparameters. Thus, for many problems we prefer to add larger batches of new labeled examples to the train set less often. Thus we seek a good batch active sampling algorithm that help select multiple unlabelled examples at once to label.

A key challenge for batch active sampling is that, for many internet application problems in particular, the pool of candidates one could label is very large and has many very similar examples. If a single criteria is used to select all the candidates in the batch, one can easily end up with a batch of very similar candidates. A good batch selection algorithm should encourage some diversity in the selected candidates.

Another important difference to the classic set-up is that in practice our candidate pool is usually changing over time, and that the candidate pool may be very large; for example, images on the web, products for sale, customer reviews, queries, people applying for a job, etc. By the time we are ready for another round of labeling, we can draw a completely different candidate pool, and the distribution of samples has likely shifted. There is generally no need to try to re-use the same fixed candidate pool for future active sampling iterations; too much keeps changing.

In light of these practical issues, our problem set-up is to do well at one-round of batch active sampling: we select one relatively large batch $B$ of candidates to label from a much larger candidate pool $\mathcal{Z}$, re-train with the new $B$ labeled data, and measure the accuracy on a held-out test set. For example, we may have a model already trained on 100,000 examples, and we expect to need to select $B=10,000$ actively-sampled candidates from a pool $\mathcal{Z}$ of 1 million unlabelled candidates to label. Once we have the $B$ newly labeled examples, it will likely take weeks to re-optimize the model, run the necessary tests, and get feedback from concerned parties before launching the revised model.

In this paper, we propose a variant of min-margin adapted to the batch setting, we analyze different active sampling algorithms for this setting on benchmark datasets, and then present two realworld case studies. We conclude with a discussion of the challenges for applying and deploying active sampling methods at scale. 


\section{CAN ONE BEAT MARGIN SAMPLING?}

A key strategy for active sampling is to select candidates that the model is most uncertain about [2]. For discriminant-based classifiers like logistic regression or neural networks, this principle is known as margin sampling [3], and selects candidates closest to the decision boundary in terms of their discriminant score. Despite many research papers in the last twenty years, margin sampling is very hard to beat experimentally [4-8].

When required to select a batch of $B$ candidates, margin sampling simply chooses the $B$ examples closest to the decision boundary, but because there is no mechanism to ensure diversity, if there is a large candidate pool to choose from, margin sampling can choose too many redundant points from high-density regions close to the initial decision boundary.

To address this, we have developed a simple algorithm that extends the core idea of margin sampling to a set of bootstrap models that increase the diversity of the selected batch.

Compared to other active sampling methods that aim to increase diversity, there are two key differences to our method: (i) our notion of diversity is supervised by the model uncertainty rather than relying on unsupervised notions of diversity like clustering, and (ii) our algorithm scales linearly in the candidate set size $|\mathcal{Z}|$ and logarithmically in the batch size $B$. Even with our proposed default hyperparameters, we show through extensive experimentation on benchmark and real datasets that the proposed bootstrapped min-margin sampling consistently performs better than margin for larger batches, and that min-margin consistently performs as well or better than other diversity-increasing active sampling methods.

\section{PRELIMINARIES}

One has an initial sample of $N$ labeled training examples $\mathcal{T}_{0}=$ $\left\{\left(x_{i}, y_{i}\right)\right\}$ for $i=1, \ldots, N$ where $x_{i} \in \mathbb{R}^{D}$ and $y_{i} \in \mathbb{N}$. Let $N_{g}=$ $\sum_{i=1}^{N} I_{y_{i}=g}$ be the number of initial examples labeled class $g$, for classes $g \in \mathbb{N}$. We also assume a candidate sample set $\mathcal{Z}$ of examples $z \in \mathbb{R}^{D}$ that can be chosen to be labeled. The goal is to select $B$ candidate examples from $\mathcal{Z}$ to add to the train set $\mathcal{T}_{0}$ to most increase the test accuracy. Given any training set $\mathcal{T}$, a learning procedure $H$ returns a model $h:=H(\mathcal{T})$, where $h(z ; g)$ is the $g$ th class discriminant function for an input $z \in \mathbb{R}^{D}$. For example, if using a neural network, $h:=H(\mathcal{T})$ would produce a trained neural network and $h(z ; g)$ could be the softmax probability of class $g$ for example $z$.

We build on margin sampling [3], which is a popular and wellregarded active learning strategy that scores candidates based on their margin:

$$
\operatorname{margin}(h, z)=h\left(z ; \hat{y}_{1}(z)\right)-h\left(z ; \hat{y}_{2}(z)\right)
$$

where $\hat{y}_{1}(z)$ and $\hat{y}_{2}(z)$ are the highest and second-highest scoring classes under predictor $h$ :

$$
\hat{y}_{1}(z):=\arg \max _{g} h(z ; g), \quad \hat{y}_{2}(z):=\arg \max _{g, g \neq \hat{y}_{1}(z)} h(z ; g) .
$$

The $B$ candidates with the smallest margins are selected as the batch to label.

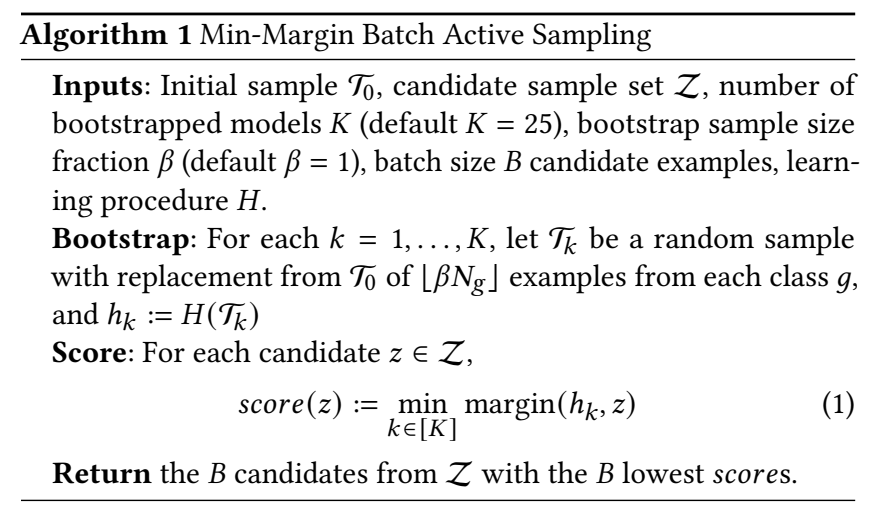

\section{MIN-MARGIN BATCH ACTIVE SAMPLING}

We propose a variant of min-margin for the batch setting we term min-margin in Algorithm 1. First, train multiple models on $K$ stratified bootstrap samples of a fraction $\beta$ of the training data one has, then select candidate examples that have the smallest margin with respect to any of the bootstrapped models. We recommend default values of $K=25$ bootstraps and $\beta=1$ as good rules of thumb for bootstraps [9], and use those for all experiments except in Section 5.6 where we analyze those choices.

\subsection{Related Work}

As noted in a 2013 survey by Fu, Zhu and Li [7], two key goals in active sampling research are to select candidates whose label is uncertain, and to try to select diverse candidates. Many active sampling methods have been devised that explicitly try to increase the diversity of the candidates. A number of methods penalize candidates based on their similarity to candidates already selected for the batch [10-13]. These methods are computationally un-appealing for large datasets and batch sizes as they scale at least $O\left(|\mathcal{Z}| \cdot B^{2}\right)$ in batch size $B$ and candidate set size $|\mathcal{Z}|$ while our proposal is $O(|\mathcal{Z}| \cdot \log B)$. A later independent study [8] did not show clearly better results from adding such diversity criteria [13] to margin sampling. Submodularity can be used to jointly select a set of diverse points close to the margin $[14,15]$. Other methods use an explicit clustering step to improve diversity $[10,16]$, or combine the margin with an empirical density estimate of the sample to obtain a density-weighted version of margin in order to encourage diversity [17, 18]. Cortes et al. [19] proposed adaptively allocating selected samples to given or random regions of the input space. These methods impose an unsupervised notion of diversity on the problem, which may help or may hurt performance. Active learning with small pools is a variant of margin sampling that only considers a small subset pool of a large candidate pool for each selection [20], it provides faster (but not better) results than margin.

Our proposal is related to Query By Committee (QBC) [21] in that both use multiple models, and a common version of $\mathrm{QBC}$ also creates multiple models by using bootstrap samples of the labeled training data [22]. QBC algorithms differ in that they score candidates by some sort of vote [22-24]. Sadly, recent results show that $\mathrm{QBC}$ does not provide any conclusive advantage over margin sampling [6]. Similarly, an experimental comparison [8] of active 

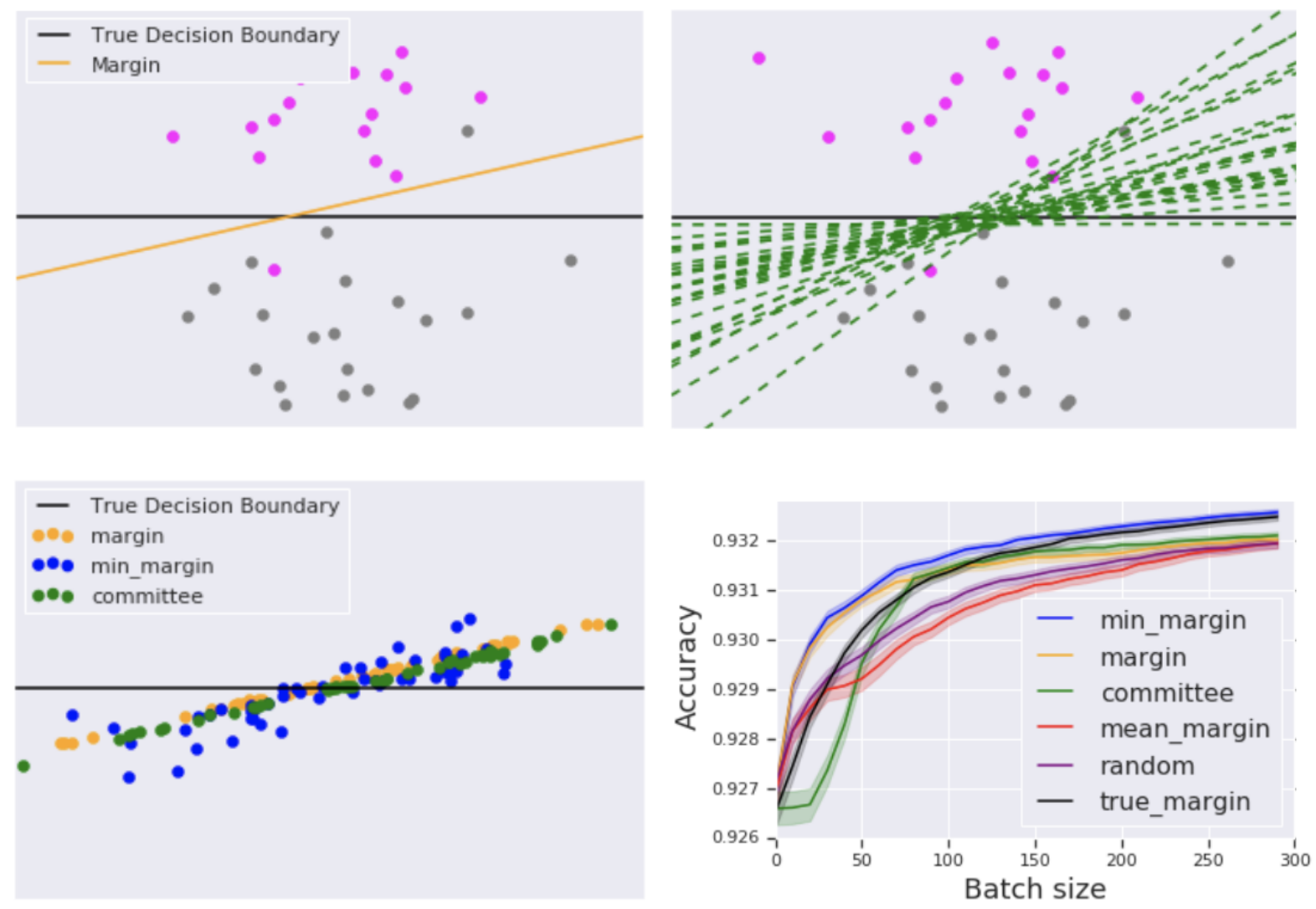

Figure 1: Figures illustrating how the min-margin algorithm acts compared to margin and QBC for a two-dimensional Gaussian simulation. See Section 4 for details.

sampling methods using SVMs on multiple remote sensing problems found margin sampling worked as well or better than the QBC variant considered (normalized entropy query by bagging [24]). We believe this is because maximizing a measure of disagreement still leads to a narrow band of highest-scoring candidates, as we will illustrate in Fig. 1. Another related idea for batch active learning with SVMs is choosing a balanced number of examples from the margins of multiple decision boundaries, and performing clustering and choosing the cluster centers as the query points [25]; however, no study has shown that this outperforms margin.

Other papers have specifically focused on the setting of batch active learning. One line of work chooses the batch by the maximum reduction in the Fisher information matrix for linear and SVM classifiers [26, 27]. Other approaches involve using kernel clustering to choose the batch for the SVM classifier [28], leveraging sub-modular optimization [29-31], adding a penalty to ensure the sampled batch matches the source distribution [32,33], maximizing the likelihood function on labeled data and minimizing the uncertainty on unlabeled examples [34].

\subsection{Understanding Min-Margin: An Illustrative Simulation}

We illustrate how min-margin sampling enhances diversity with a simulation where samples from each class are drawn IID from a Gaussian with univariate covariance but different means for the two different classes. The initial train set is 40 IID examples, shown for the two classes as pink/bright and gray/dull dots in Fig. 1 top left. A logistic regression model trained on these produces the yellow decision boundary, far from the black true decision boundary.

We bootstrap $K=25$ new models on the 40 train samples, their 25 decision boundaries are shown as green dashed lines in Fig. 1 top right. We randomly draw a candidate pool of 8,000 IID samples to actively-sample from, and a random IID test set of 10,000 samples. Then we actively sample batches of different sizes $B$ using margin, the proposed min-margin, and query by committee (QBC) [22]. For each method and batch size $B$, we re-train on the union of the initial train set and that method's selected batch, and measure the test accuracy.

For $B=50$ the selected candidates for each method are shown in Fig. 1 bottom left. Margin's choices, in yellow, all lie very close to the initial decision boundary. However, this decision boundary can be highly biased. Thus, margin sampling can fail to choose points in critical regions necessary to learn the true decision boundary, 
even if $B$ is large. For fixed $B$, the larger the candidate set $\mathcal{Z}$ is, the more narrow the region of candidates chosen by margin sampling.

Committee algorithms that score based on a notion of maximal disagreement of the bootstrap models will have a similar lack of diversity. QBC's [22] top choices, in green, similarly all lie within a narrow double-cone-shaped region where the bootstrapped decision boundaries maximally disagree. In contrast, min-margin picks candidates (in blue) that are near any of the 25 bootstrapped margins, giving it a supervised diversity throughout the uncertainty region defined by the bootstrapped margins.

Further, if the candidate set $\mathcal{Z}$ is very large, QBC is likely to pick up candidates in sparse regions where classifier disagreement is high, but where improving the classifier is less important because so few examples will be affected by any improvement in the sparse parts of the feature space. In fact, one sees in Fig. 1 bottom left that QBC top $B=50$ choices (in green) are the furthest out on both sides - with exponentially lower probability from the Gaussians. This problem is compounded in higher-dimensional spaces where much of the maximal-disagreement area is sparse. In contrast, this is less of a problem for min-margin, because the bootstrap decision boundaries will tend to diverge more in sparser regions (as seen in Fig. 1 top right), and so candidates in sparse regions are less likely to be near any bootstrap decision boundary, and are less likely to be picked up.

After re-training with the new batch, the test results are shown in Fig. 1 bottom right, averaged over 500 random draws of the simulation, with confidence intervals marked by shaded regions. Min-margin achieves the best test accuracy for all batch sizes, and as batch size $B$ grows (and candidate pool $\mathcal{Z}$ was held fixed), minmargin win over margin increases, as we had hoped. The differences in test accuracy are small for this tiny easy problem, but importantly it shows that in a well-controlled standard Gaussian simulation the min-margin algorithm is best.

We also compared to pure random sampling, which picks candidates that are too diverse and do not help the model, and to sorting by the mean of the bootstrapped models' margins, which as similar problems as described above for QBC.

We also compare to the oracle approach of sorting the candidates with respect to the true margin to the true decision boundary, which can only be run because this is a simulation with known class-conditional distributions. Remarkably, min-margin even outperforms margin sampling with the true margin, and is the only method to beat the true margin for all batch sizes. True margin performs rather badly for small batch sizes $B$ because one most needs new examples that can fight the confusions caused by the initial sample $\mathcal{T}_{0}$, but true margin does not take $\mathcal{T}_{0}$ into account at all. The larger the batch size $B$, the less important the initial sample $\mathcal{T}_{0}$ is, and the better true margin performs.

In fact, a striking property of the added diversity of min-margin is that it adapts to the uncertainty of the initial sample. If the initial sample is noisy, then the set of bootstrapped models will also likely have high variability, and a more diverse set of candidates will be selected.
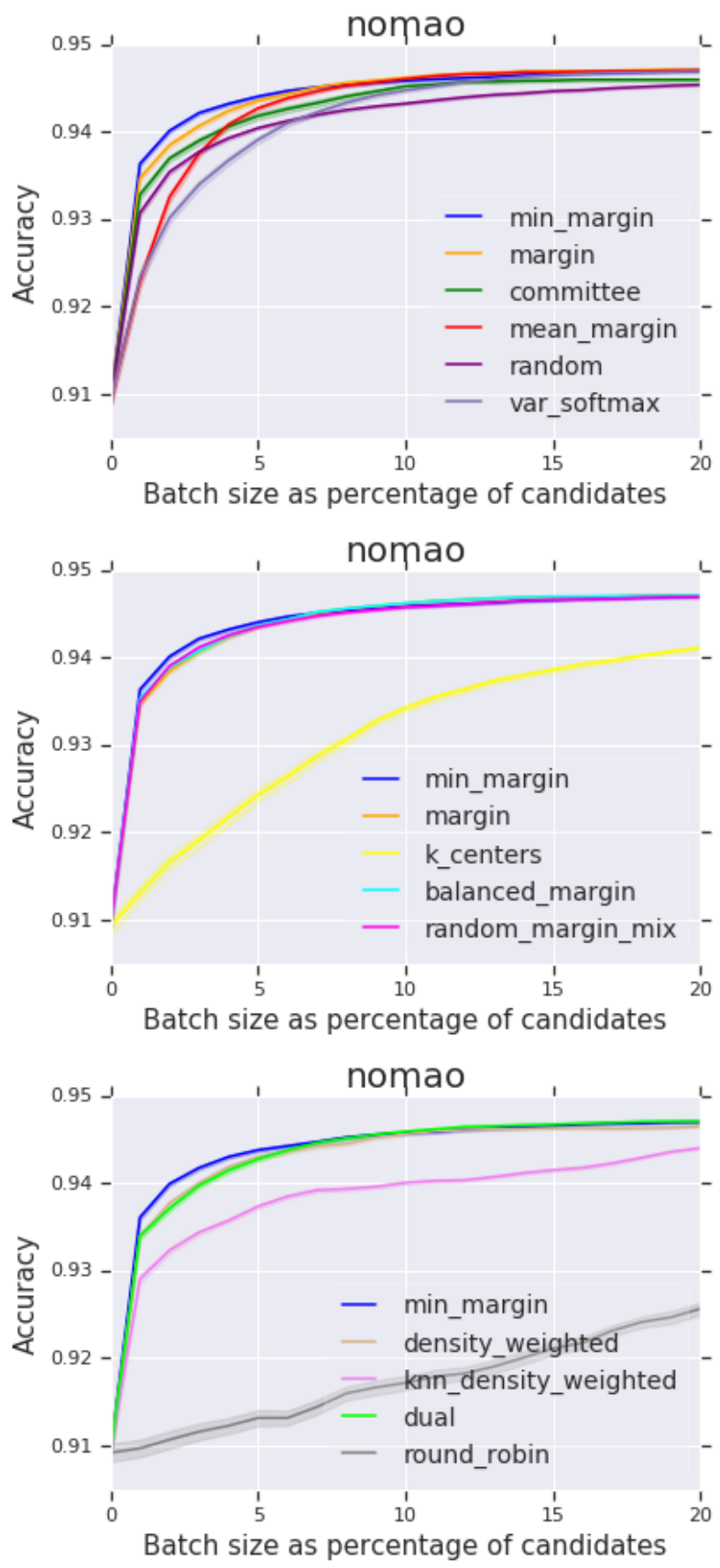

Figure 2: Test accuracies vs. the batch size for nomao UCI benchmark dataset. The standard error is shaded.

\section{BATCH ACTIVE SAMPLING EXPERIMENTS}

We compare the proposed min-margin sampling algorithm to twelve other active sampling strategies on 7 benchmark datasets, showing the effect of different batch sizes, and for both logistic regression and neural network classifiers. 

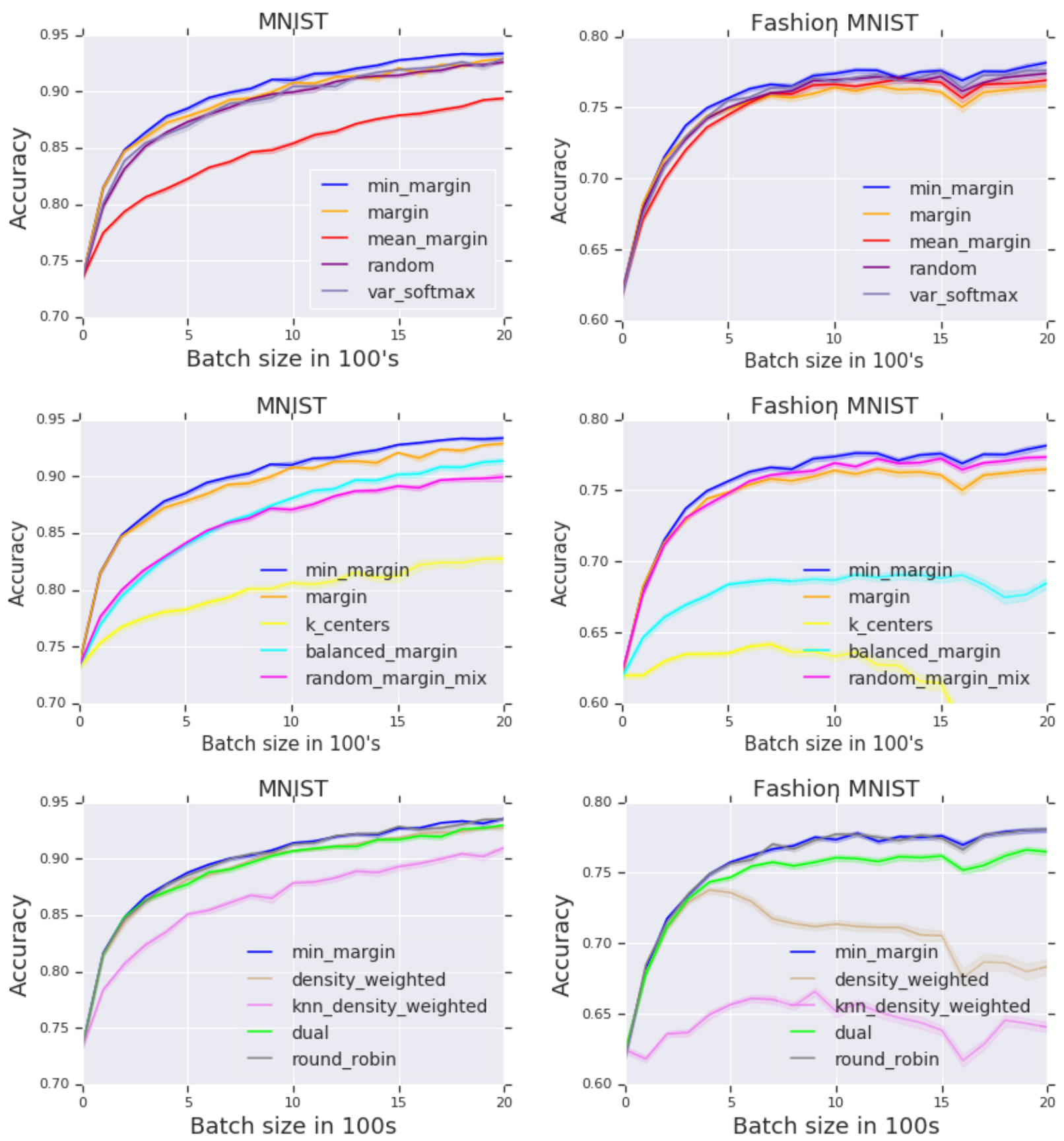

Figure 3: Left: MNIST results. Right: Fashion MNIST Results. Shown are the test accuracies vs. the batch size of the selected active samples, and the standard errors over the 100 runs is shaded. For both datasets, min-margin is one of the best performing methods for every batch size. The different plots compare different subsets of methods.

\subsection{Real world Issues}

We attempted to make our benchmark dataset experiments similar to our real-world problem the best we could. However, in the realworld, the candidate pools are generally much larger than we can mimic here (because for benchmark experiments we are limited to examples already labeled), and in practice our candidate pool distribution is generally shifting between rounds of active-sampling and re-training, which for today's large models can take days or weeks. Batch sizes sent to human raters for labeling may also be quite large to simplify processing. In order to run experiments that give 


\begin{tabular}{llllllll}
\hline & MNIST & Fash. MNIST & nomao & magic04 & shuttle & a9a & cod-rna \\
\hline margin & 88.89 & 74.49 & 94.31 & 77.55 & 95.04 & 83.93 & 93.04 \\
QBC & n/a & n/a & 94.18 & 78.74 & 94.66 & 83.83 & 92.87 \\
mean-margin & 84.52 & 74.57 & 94.19 & 75.83 & 94.54 & 83.90 & 92.99 \\
random & 88.40 & 74.94 & 94.05 & 78.45 & 94.40 & 83.79 & 92.30 \\
var-softmax & 88.55 & 75.09 & 94.05 & 78.60 & 95.22 & 83.90 & 92.42 \\
k-centers & 79.77 & 61.77 & 93.06 & 76.47 & 84.04 & 83.46 & 91.45 \\
balanced margin & 86.27 & 67.83 & $\mathbf{9 4 . 3 2}$ & 77.56 & 94.66 & 83.94 & 93.04 \\
rand-margin mix & 85.78 & 74.96 & 94.29 & 79.14 & 94.29 & 83.89 & 92.81 \\
dense-weight & 88.97 & 70.28 & 94.24 & 79.01 & 94.05 & $\mathbf{8 3 . 9 5}$ & 92.55 \\
knn dense-weight & 86.19 & 64.41 & 93.78 & 78.13 & 89.61 & 83.70 & 91.45 \\
dual & 88.94 & 74.39 & 94.27 & 77.55 & 94.97 & 83.95 & 93.07 \\
round-robin & 89.49 & 75.54 & 91.71 & 74.79 & 91.79 & 83.76 & 84.54 \\
min-margin & $\mathbf{8 9 . 5 4}$ & 75.57 & $\mathbf{9 4 . 3 2}$ & 78.83 & $\mathbf{9 5 . 3 9}$ & $\mathbf{8 3 . 9 5}$ & $\mathbf{9 3 . 0 7}$ \\
\hline
\end{tabular}

Table 1: Area Under the Curve (accuracy vs. batch size) for the curves shown in Fig. 2, Fig. 3, and Fig. 6 in the Supplemental. Highest score on each dataset is bold and green, 2nd and 3rd highest scores in orange.

us insight into how batch active sampling methods can be expected to compare in these real-world settings, all of our experiments are like the Gaussian simulation above in that they actively sample only one batch once, re-train with the initial plus batch train sets, and report the test metrics. This enables more realistic experiments in terms of the ratio of the batch size to the candidate pool, which better highlights the real-world diversity problems with standard margin sampling. Further, this one-round setting allows an experimentally cleaner use of the limited experimental candidate pool than the traditional active sampling set-up where one performs many iterations of active sampling selected from the same fixed candidate pool.

\subsection{Comparison Methods}

We compare min-margin against the following twelve methods (details in the Supplemental):

1. margin: standard margin sampling

2. committee: $\mathrm{QBC}[22]$

3. mean-margin: score candidates by their mean-margin over the ensemble (lower is better)

4. random: uniform random sampling of the candidate set without replacement

5. var-softmax: score candidates by their margin-variance over the ensemble (higher is better)

6. k-centers: sampling according to the $k$-centers coresets method to enhance diversity of the samples [35]

7. balanced-margin: balances the margin score with a penalty on pairwise diversity [11]

8. random-margin-mix: 50-50 mix of random and margin; a common strategy to enhance diversity

9. density-weighted: score candidates by the product of margin and density estimate to enhance diversity [17]

10. knn-density-weighted: similar to density-weighted, but uses $k$-NN density estimator to enhance diversity [18]

11. dual: scores candidates by a weighted sum of margin and a density-weighted margin to enhance diversity [36]

12. round-robin: a variant of our proposed min-margin that we considered we select round $(B / K)$ examples from each of the $K$ bootstrapped margins.

All methods that use bootstrap models used the same set of $K=$ 25 bootstrapped models with $\beta=1$ re-sampling. With these recommended default choices, all compared methods are hyperparameterfree unless otherwise noted.

\subsection{MNIST and Fashion MNIST datasets}

We use an initial sample size of 100 for each problem. For MNIST, we use a neural network with two hidden layers each with 512 units and ReLU activations. All experiments trained with ADAM's default stepsize .001 for 100 epochs. For Fashion MNIST, we used one hidden layer with 128 units (as per the Keras tutorial). Results in Fig. 3 were averaged over 100 different random splits between initial samples and candidate samples, and tested on the standard test sets.

\subsection{Benchmark UCI Datasets}

We also compared on five benchmark UCI datasets that are popular for testing active sampling methods: nomao (118 features, 34,465 datapoints), shuttle (9 features, 43,500 datapoints), magic04 (10 features, 19,020 datapoints), a9a (123 features, 32,561 datapoints) and cod-rna (8 features, 59,535 datapoints). We used logistic regression as the learner, trained using scikit learn's implementation [37] under default settings. Test set accuracy was averaged over $100 \mathrm{random}$ initial/candidate/test splits of each dataset, where the initial sample size is 100 and candidate/test sets are of equal size.

\subsection{Benchmark Experiment Results}

Fig. 3 shows that min-margin is the best strategy on both MNIST and Fashion MNIST against 11 methods (we did not run the QBC comparison for the multi-class experiments).

Table 1 gives the area under the curve of the charts in Fig. 3 and for the analogous test accuracy vs batch size charts for the UCI benchmark datasets (full charts in the Supplemental). Min-margin is the best (or tied for the best) on 6 of the 7 datasets, and is 3rd best 

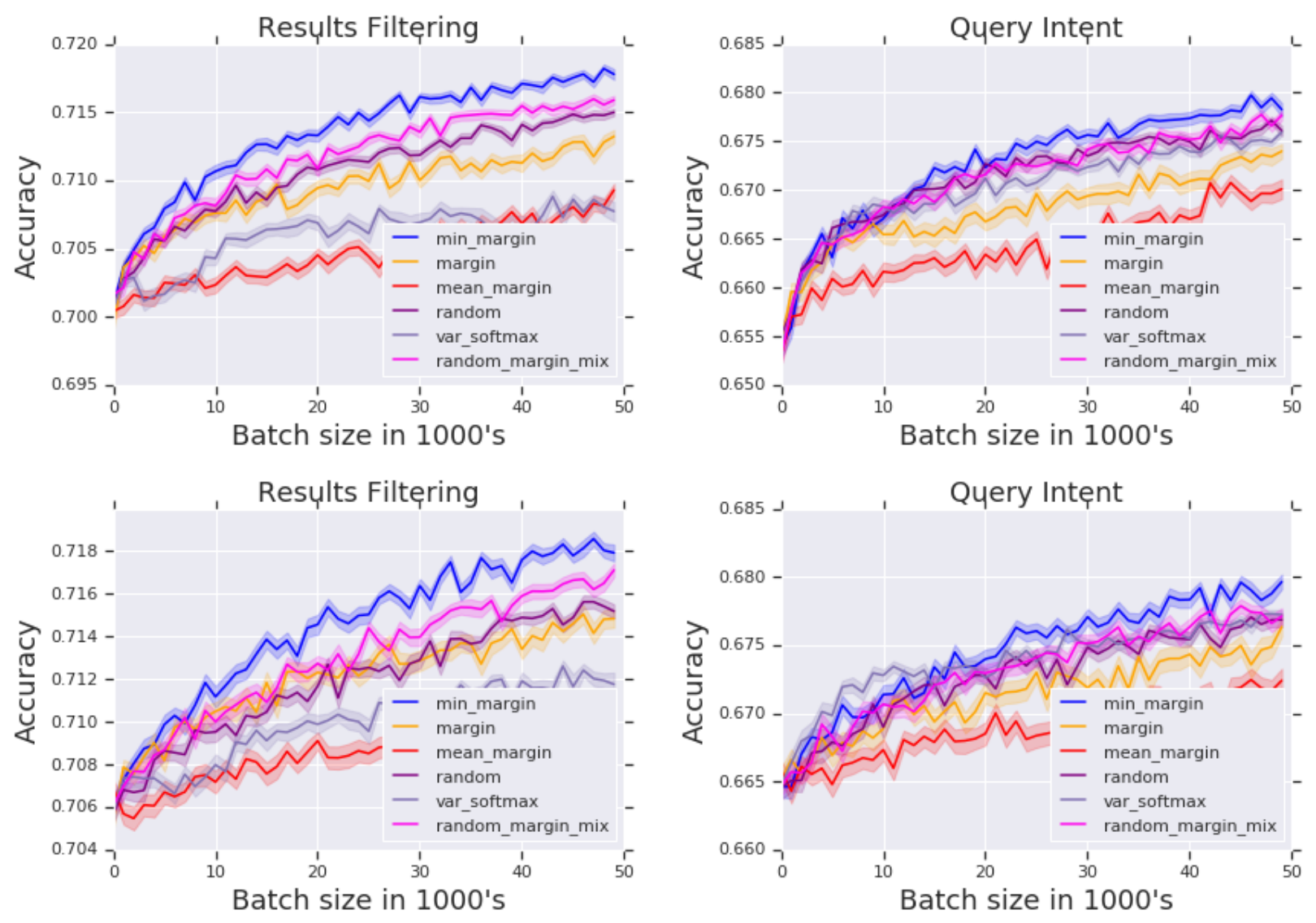

Figure 4: Test accuracies vs. the batch size. The standard error is shaded. Top Row: Initial models trained on 5, 000 examples. Bottom Row: Initial models trained on 10, 000 examples.

on the remaining dataset. None of the other algorithms performs as consistently well.

In fact, min-margin is better than margin on all 7 problems. None of the other algorithms beats margin on more than 3 problems. Margin sampling is a hard baseline to improve upon.

The round-robin variant of the proposed min-margin works almost as well as min-margin on the MNIST and Fashion MNIST datasets, but not as well on the UCI benchmark datasets.

Going back to Fig. 3 and the analogous curves for the UCI datasets, the wins for min-margin over margin tend to get bigger as the batch size gets bigger. This effect is most pronounced for the Fashion MNIST (compare the orange to blue lines). Of course, at some point all the useful training samples are picked by any of the sampling strategies, and so the results will converge together again when the batch size is a large percentage of the candidate pool.

\subsection{Robustness To Hyperparameters}

With the exception of the results in Fig. 5, all of the results in this paper used our recommended default of $K=25$ bootstrap models with $\beta=1$ bootstrap sampling, and so all experiments above were hyperparameter-free. Later, we ran a number of experiments to understand the effect of these two hyperparameters.

In the left column of Fig. 5, we show the effect of different $K$ choices as a function of the batch size. These plots and our other experiments (not shown due to space) suggested that the exact choice of $K$ is not so important as long as it is sufficiently big, and that $K=25$ was consistently good if not best. If possible, using larger $K$ is probably better, though it takes more computation, which is why we recommend a default $K=25$.

The results in the right column of Fig. 5 show the greater sensitivity we see to the size of the bootstrap sample, controlled by $\beta$. A smaller bootstrap fraction $\beta$ produces more diverse bootstrapped models [9], in turn making min-margin more like random sampling. Indeed, the smaller $\beta$ 's work best on the shuttle dataset, which is also the dataset that random sampling works relatively best on. For other datasets, like cod-rna, the opposite is true. We concluded that our default $\beta=1$ is a reasonable default choice, but there is certainly room to win more if one can validate $\beta$ as a hyper-parameter, or if randoms-sampling seems to work well for a problem, lower $\beta$. 

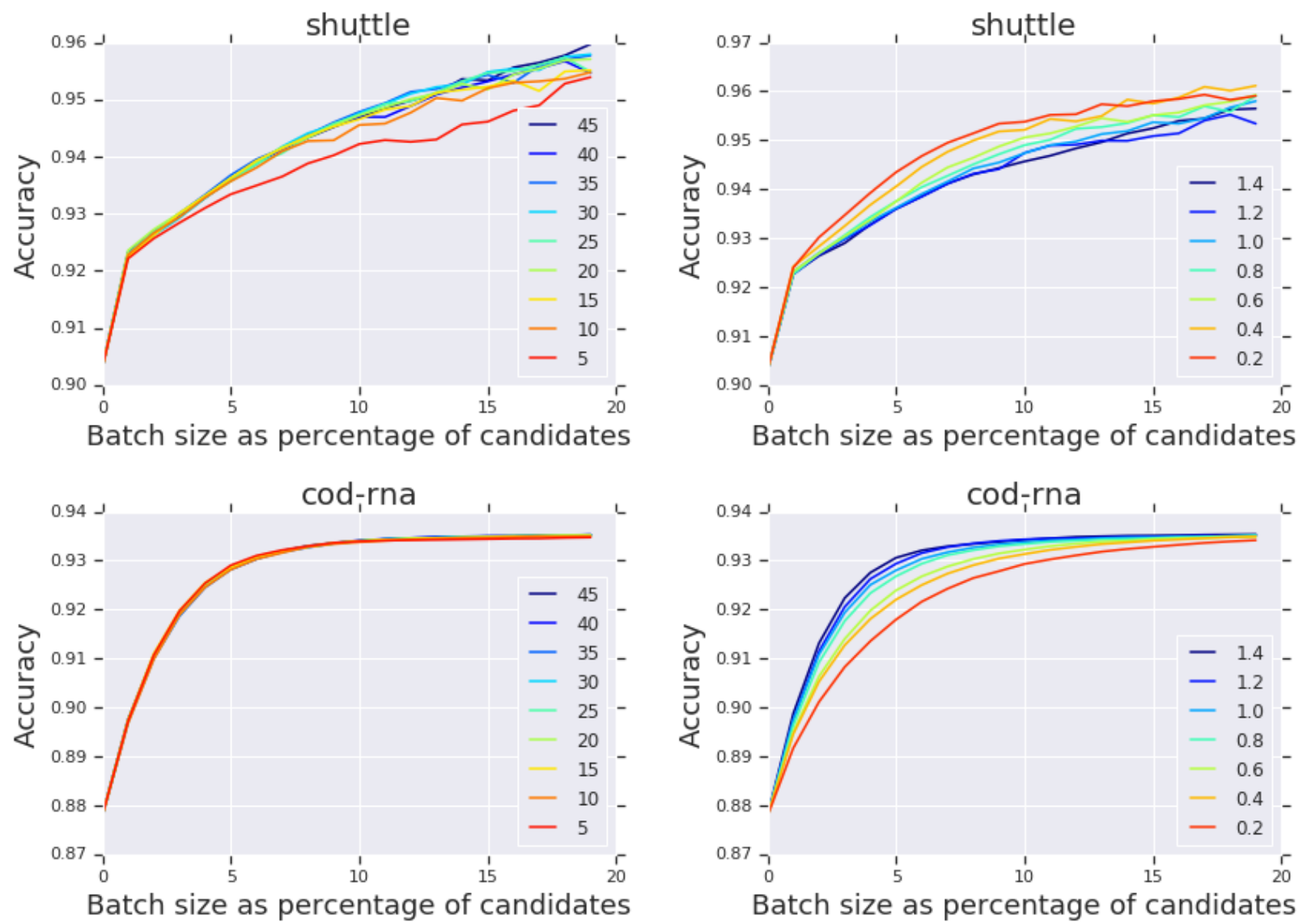

Figure 5: Left: Test accuracy of min-margin for different choices of the number of bootstrap models $K$ (with default $\beta=1$ ). Right: Test accuracy of min-margin for different choices of the fraction of the train set bootstrapped $\beta$ (with default $K=25$ ).

\section{REAL-WORLD CASE STUDIES}

In this section, we compare the performance of the proposed minmargin algorithm on two proprietary binary classification problems at Google where the problem set-up is as described in Section 1.

For these datasets, we had to drop the more computationally expensive comparison methods due to the large batch and candidate sizes, and only compared to the five margin variants, including the basic margin sampling algorithm which was the 2nd best method (in terms of how many datasets it won on when compared to any other method) on our benchmark experiments as shown in Table 1.

As in the benchmark experiments, we limit the candidate set to be a subset of examples that we already have labels for, but in practice the true candidate pool size $|\mathcal{Z}|$ would actually be orders of magnitude larger, increasing the importance of ensuring diversity. Results Filtering: The task is to classify whether a candidate result is promising enough to be worth more expensive processing or should be filtered out (16 features, 1,282,532 labeled examples total). Query Intent: The task is to classify the intent of a query between two possible intents (32 features, 420,000 labeled examples total).

For both experiments, we used a 2-layer neural network as the learner with 10 hidden units, and trained with ADAM with default
0.001 step size for 20 train epochs with mini-batch sizes of 100 . We averaged over $100 \mathrm{random}$ initial/candidate/test splits of the data.

Fig. 4 shows results for three sizes of the inital train set. The rest of the labelled examples were split evenly between the candidate set and the test set. For both models, for larger batch sizes there is a consistent statistically significant gain of roughly $+0.5 \%$ accuracy, which would be a worthwhile gain for these real-world internet problems with widespread impact.

\subsection{Major Challenges to Deployment}

This effort has reached the milestone of being considered for a dedicated budget. Each round of labeling 100,000 new examples requires a non-trivial budget allocation at upper managerial levels, and must be weighed against competing priorities.

The bottom row of Fig. 4 shows that with models trained on just 10,000 examples, paying to label a batch of $B=50,000$ samples can deliver a $1 \%$ improvement in accuracy, and would be a very good return on investment in the new labels. However, in practice, our real-world models are trained on the full labelled datasets, that is, 1,282,532 examples and 420,000 examples (although the training sets are always evolving as stale samples are tossed and new examples 
accrued). And we lack good methods to predict how much value will be gotten from batch of $B$ samples. Based on randomly subtracting examples, we expect to need at least $B=100,000$ new labeled examples to be certain of a measurable gain on the battery of tests that these models undergo for launch approval [1]. Also, it is difficult to predict what kinds of gains will be had, and blanket gains may not be considered as important as targeted gains that can be better achieved by feature engineering to cover known blind spots in the model.

\section{CONCLUSIONS}

Margin sampling is known to be hard to beat in practice, but we worried it would pick candidates that were not diverse when used for our large batches from very large candidate pools. We created a simple min-margin bootstrapped variant of margin sampling, and found it is effective at increasing the diversity of active samples in a supervised and adaptive manner. Extensive experiments (not all shown due to lack of space) showed that the proposed minmargin works consistently well across a broad set of problems, a diverse range of models, and against a broad range of baselines. Min-margin's value over margin increases for larger batch sizes.

We also compared to a number of other methods that have been proposed to increase the diversity of selected samples in other ways $[11,17,18,35,36]$ on 7 benchmark datasets. We found minmargin was consistently better than each of these (see Table 1). In practice, a popular way to get guaranteed diversity is to just mix random sampling and margin sampling. We saw that does fairly well compared to the more complex algorithms, but min-margin is almost always a bit better.

The proposed min-margin also beat a variant of itself we called round-robin, where each of the $K$ bootstrap models choose $B / K$ of the candidates, which produces even more diverse examples. However, we believe this round-robin approach interferes with the adaptive sampling over the $K$ margins that helps the selected candidates reflect the candidate pool distribution.

Overall, our analysis and extensive experimentation leads us to believe that min-margin will work robustly and efficiently for even large batch sizes and very large candidate pools.

\section{REFERENCES}

[1] Quentin Cormier, Mahdi Milani Fard, Kevin Canini, and Maya R. Gupta. Launch and iterate: Reducing prediction churn. NeurIPS, 2016.

[2] David D. Lewis and William A. Gale. A sequential algorithm for training text classifiers. ACM SIGIR, 1994.

[3] Tobias Scheffer, Christian Decomain, and Stefan Wrobel. Active hidden Markov models for information extraction. Proceedings of the International Conference on Advances in Intelligent Data Analysis (CAIDA), 2001.

[4] Oscar Reyes, Abdulrahman H. Altalhi, and Sebastián Ventura. Statistical comparisons of active learning strategies over multiple datasets. Knowledge Based Systems, pages 274-288, 2018.

[5] Andrew I. Schein and Lyle H. Ungar. Active learning for logistic regression. Machine Learning, pages 235-265, 2007.

[6] Maria E. Ramirez-Loaiza, Manali Sharma, Geet Kumar, and Mustafa Bilgic. Active learning: an empirical study of common baselines. Data Mining and Knowledge Discovery, 2016.

[7] Yifan Fu, Xingquan Zhu, and Bin Li. A survey on instance selection for active learning. Knowledge and Information Systems, pages 249-283, 2013.

[8] Devis Tuia, Michele Volpi, Loris Copa, Mikhail Kanevski, and Jordi Munoz-Mari. Survey of active learning algorithms for supervised remote sensing image classification. IEEE Journal Selected Topics in Signal Processing, pages 606-617, 2011.

[9] Brad Efron and Rob Tibshirani. An Introduction to the Bootstrap. Chapman and Hall, 1993.
[10] Zhi-Hua Zhou, Yu-Yin Sun, and Yu-Feng Li. Multi-instance learning by treating instances as non-iid samples. ICML, 2009.

[11] Klaus Brinker. Incorporating diversity in active learning with support vector machines. ICML, 2003.

[12] Atsushi Fujii, Kentaro Inui, Takenobu Tokunaga, and Hozumi Tanaka. Selective sampling for example based word sense disambiguation. Computational Linguistics, 1998.

[13] Marin Ferecatu and Nozha Boujemaa. Interactive remote sensing image retrieval using active relevance feedback. IEEE Trans. Geosciences and Remote Sensing, pages 818-826, 2007.

[14] Kai Wei, Rishabh Iyer, and Jeff Bilmes. Submodularity in data subset selection and active learning. ICML, 2015.

[15] Steven C. H. Hoi, Rong Jin, Jianke Zhu, and Michael. R. Lyu. Batch mode active learning and its application to medical image classification. ICML, 2006.

[16] Zuobing Xu, Ram Akella, and Yi Zhang. Incorporating diversity and density in active learning for relevance feedback. Proceedings of the European Conference on IR Research (ECIR), 2007.

[17] Hieu T. Nguyen and Arnold Smeulders. Active learning using pre-clustering. ICML, 2004.

[18] Jingbo Zhu, Huizhen Wang, Benjamin K Tsou, and Matthew Ma. Active learning with sampling by uncertainty and density for data annotations. IEEE Transactions on audio, speech, and language processing, 18(6):1323-1331, 2009.

[19] Corinna Cortes, Giulia DeSalvo, Claudio Gentile, Mehryar Mohri, and Ningshan Zhang. Region-based active learning. In AISTATS, pages 2801-2809, 2019.

[20] Seyda Ertekin, Jian Huang, Leon Bottou, and C. Lee Giles. Learning on the border: Active learning in imbalanced data classification. Proc. CIKM, 2007.

[21] H. Sebastian Seung, Manfred Opper, and Haim Sompolinsky. Query by committee. COLT, 1992.

[22] Naoki Abe and Hiroshi Mamitsuka. Query learning strategies using boosting and bagging. ICML, 1998.

[23] Ido Dagan and Sean P. Engelson. Committee-based sampling for training probabilistic classifiers. ICML, 1995 .

[24] Loris Copa, Devis Tuia, Michele Volpi, and Mikhail Kanevski. Unbiased query by bagging active learning for VHR image classification. Proc. SPIE Remote Sens. Conf.

[25] Swarnajyoti Patra and Lorenzo Bruzzone. A batch-mode active learning technique based on multiple uncertainty for svm classifier. IEEE Geoscience and Remote Sensing Letters, 9(3):497-501, 2011.

[26] Steven CH Hoi, Rong Jin, Jianke Zhu, and Michael R Lyu. Batch mode active learning and its application to medical image classification. In Proceedings of the 23rd international conference on Machine learning, pages 417-424, 2006.

[27] Steven CH Hoi, Rong Jin, and Michael R Lyu. Large-scale text categorization by batch mode active learning. In Proceedings of the 15th international conference on World Wide Web, pages 633-642, 2006.

[28] Begüm Demir, Claudio Persello, and Lorenzo Bruzzone. Batch-mode activelearning methods for the interactive classification of remote sensing images. IEEE Transactions on Geoscience and Remote Sensing, 49(3):1014-1031, 2010.

[29] Yuxin Chen and Andreas Krause. Near-optimal batch mode active learning and adaptive submodular optimization. In International Conference on Machine Learning, pages 160-168. PMLR, 2013.

[30] Steven CH Hoi, Rong Jin, Jianke Zhu, and Michael R Lyu. Semisupervised SVM batch mode active learning with applications to image retrieval. ACM Transactions on Information Systems (TOIS), 27(3):1-29, 2009.

[31] Shayok Chakraborty, Vineeth Balasubramanian, and Sethuraman Panchanathan. Adaptive batch mode active learning. IEEE transactions on neural networks and learning systems, 26(8):1747-1760, 2014.

[32] Zheng Wang and Jieping Ye. Querying discriminative and representative samples for batch mode active learning. ACM Transactions on Knowledge Discovery from Data (TKDD), 9(3):1-23, 2015.

[33] Rita Chattopadhyay, Zheng Wang, Wei Fan, Ian Davidson, Sethuraman Panchanathan, and Jieping Ye. Batch mode active sampling based on marginal probability distribution matching. ACM Transactions on Knowledge Discovery from Data (TKDD), 7(3):1-25, 2013.

[34] Yuhong Guo and Dale Schuurmans. Discriminative batch mode active learning. In NIPS, pages 593-600. Citeseer, 2007.

[35] Ozan Sener and Silvio Savarese. Active learning for convolutional neural networks: A core-set approach. ICLR, 2018.

[36] Pinar Donmez, Jaime G Carbonell, and Paul N Bennett. Dual strategy active learning. In European Conference on Machine Learning, pages 116-127. Springer, 2007.

[37] Fabian Pedregosa, Gaël Varoquaux, Alexandre Gramfort, Vincent Michel, Bertrand Thirion, Olivier Grisel, Mathieu Blondel, Peter Prettenhofer, Ron Weiss, Vincent Dubourg, Jake Vanderplas, Alexandre Passos, David Cournapeau, Matthieu Brucher, Matthieu Perrot, and Édouard Duchesnay. Scikit-learn: Machine Learning in Python. fournal of Machine Learning Research, 12:2825-2830, 2011. 


\section{A SUPPLEMENTAL: MORE DETAILS ON COMPARISON METHODS AND TRAINING}

All methods that use the bootstrapped models used the same set of bootstrapped classifiers. All compared methods are used under their default settings (for the bootstrap models, we used our recommended default of $K=25$ bootstrap models, and $\beta=1$ bootstrap fraction). We now give a description of the additional baselines used:

- balanced-margin. This is the method of Brinker (2003) [11] which adds to the objective a normalized term to encourage diversity. It selects a batch by iteratively choosing the candidate that minimizes $\lambda \cdot\left|g\left(x_{i}\right)\right|+(1-\lambda) \max _{j \in S} k\left(x_{i}, x_{j}\right)$ where $S$ is the set of indices of the examples chosen thus far and adding it to the set $S$ until the batch size is reached and $k$ is a normalized distance kernel. We use their default setting of $\lambda=0.5$ and use the cosine similarity as $k$.

- k-centers. This is a method presented by Sener and Savarese (2018) [35] that selects the batch using the greedy $k$-centers algorithm (i.e. setting $k$ to be the size of the batch and the centers would be the examples to query for labels). As a reminder, the $k$-centers objective is to find $k$ centers that minimizes the maximum distance from any example to its closest center. We use cosine similarity as the distance metric. We note that [35] provides a robust version of $k$-centers which they show performs marginally better than $k$-centers. We use the basic version which has no hyperparameters to tune. We note however that [35] designed this method for convolutional neural networks, while in our experiments, none were convolutional neural networks.

- random-margin-mix. This method chooses half the examples based on margin and the other half randomly.

- density-weighted: This is the method of Nguyen et al (2004) [17] which scores examples by the product of the margin and a density estimate. We borrow the implementation from https://github.com/ntucllab/libact/tree/master/libact/query_ strategies which implements the density estimate by using $K$-means on all the examples and an example's density would be the number of examples in its corresponding cluster. We set $K=10$ and also use dimensionality reduction with PCA down to 10-dimension for speed considerations. Then examples are selected with minimum product of margin and density.

- knn-density-weighted: This is the method of Zhu et al. (2009) [18], which is similar to density-weighted, but uses the $k$-nearest neighbor density estimator. We set $k=100$ and also PCA down to 10 dimensions for efficiency.

- dual: This is the method of Donmez et al. (2007) [36], which scores examples based on a weighted sum of margin and a density-weighted margin. If $\alpha$ is the mean margin score for all the examples, then the examples are scored as follows: $(1-\alpha) \cdot$ margin $+\alpha \cdot$ density, where density is computed in the same way as in the density-weighted baseline.

We show additional charts comparing these methods. We found that these methods were often not competitive with many of the baselines shown in the main text. We show these for the benchmark datasets and MNIST and Fashion MNIST. For the real-world case studies, balanced-margin and k-centers were computationally infeasible due to the size of the datasets so we don't show the comparisons for those.

\section{A.1 Additional Experiments: UCI Benchmark datasets}

On the next page are the remaining plots for the UCI datasets in Figure 6. 

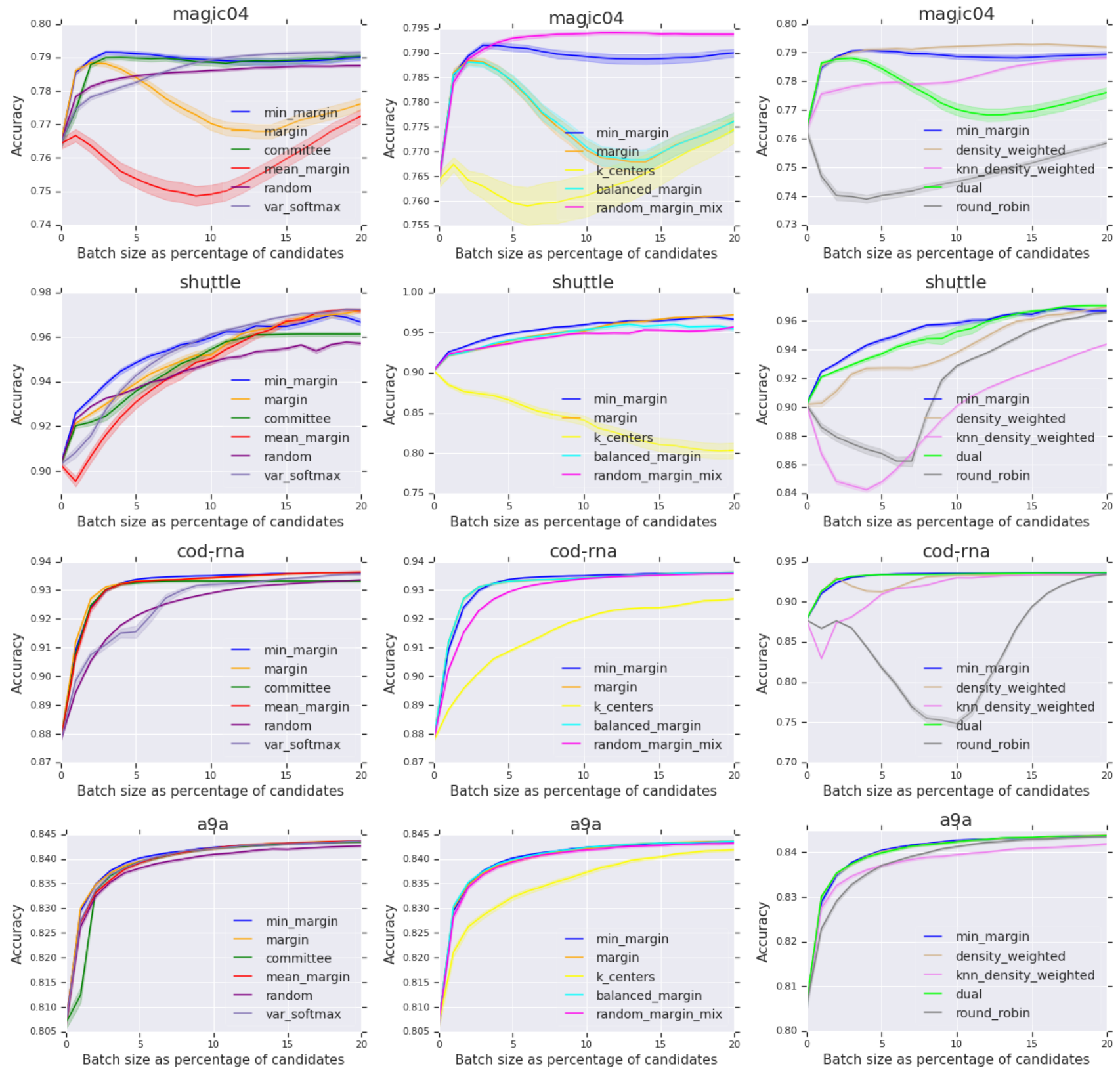

Figure 6: Test accuracies vs. the batch size for UCI benchmark datasets. The standard error is shaded. 